\title{
ESPACIOS GEOGRÁFICOS Y LOCALIZACIONES EPISTEMOLOGICAS: LA RATIO ENTRE LA LOCALIZACIÓN GEOGRÁFICA Y LA SUBALTERNIZACIÓN DE CONOCIMIENTOS
}

\author{
Walter D. Mignolo* \\ Universidade de Duke, Estados Unidos
}

Las reflexiones que siguen sobre el espacio y la localización comenzaron en un seminario que dicté en Duke University en el semestre de primavera (enero a abril) de 1996 y que tuvo una primera formulación concisa en el Congreso de Literaturas Comparadas, celebrado en Rio de Janeiro en Agosto de 1996. Sin embargo, la problemática general había comenzado mucho antes. Y esta problemática general era la ecuación entre legados coloniales y teorización poscolonial que no sólo ganaba terreno en la academia norteamericana, sino que también se escribía en inglés, sobre la experiencia paradigmática del colonialismo del "Commonwealth". En la medida en que Gayatri Spivak y Homi Bhabha se tomaron, particularmente en U.S., como "token" de la teoría poscolonial (cuestión que por cierto no inculpa a los mencionados, sino al sistema de mercantilización de los conocimientos y a la subyacente idea de desarrollo que equipara nuevas ideas con nuevos modelos de automóviles), se procesaba la reflexión poscolonial como una tendencia crítica en los estudios literarios y culturales, más que

\footnotetext{
"Walter D. Mignolo. Argentino, professor da Universidade de Duke (EUA), onde coordena os programas de Literatura latino-americana e Antropologia Cultural. Editor da revista Dispositio/n e da nova série "Latin America Otherwise". Destaca-se entre suas publicações em português o livro "Histórias Locais/ Projetos Globais: Colonialidade, saberes subalternos e pensamento liminar" (Belo Horizonte: Ed. UFMG, 2003), resenhado neste número por Valter do Carmo Cruz.
} 
como el comienzo de un desplazamiento paradigmático importante (Mignolo, 1996). El problema era aquí que, al concebirse así la reflexión crítica poscolonial, se la concebía a contramano de lo que la teorización poscolonial (a la manera de Frantz Fanon, Amilcar Cabral, Aimé Césaire, Edouard Glissant), pretendería hacer. Esto es, el proyecto de la teoría poscolonial, tal como la entendía y la entiendo, era paulatinamente consumido por el proyecto epistemológico del cual la teorización poscolonial trataría de escapar (Mignolo, 1996). Mi interés por la colonización y la teorización poscolonial (no, claro está, para decidir cual país es poscolonial y cual no) no era, no fue, ni es la de un fiel converso que trata de convertir a otros a su doctrina, sino la de un observador de cierto escenario intelectual contemporáneo y la de un participante comprometido en la reflexión crítica sobre los legados coloniales que continúan marcando pautas en la interpretación de los fenómenos culturales, en las políticas estatales y en los conflictos internacionales.

La reflexión crítica sobre el colonialismo (occidentalismo, orientalismo) no afecta sólo a los países del Tercer Mndo, sino a todo el planeta. Después de 1947, es tan poscolonial India como Inglaterra; después de 1962, tanto Algeria como Francia; despues de 1898, tanto Cuba como España. Pero, también, después de 1898, tanto Cuba como Puerto Rico entran en un nuevo tipo de relaciones coloniales que las diferencia de los otros países hispanoamericanos. El hecho de que haya todavía situaciones coloniales, no hace mella en el hecho de que podamos elaborar una reflexión crítica sobre el colonialismo tanto con miras académicas como de transformación social. Si la reflexión crítica sobre el colonialismo y los legados coloniales hoy se debe llamar poscolonialismo me importa, en realidad, poco. En cuanto a cuestión de nombres, tiendo cada vez más a hablar de posoccidentalismo, puesto que la occidentalización es la preocupación que se registra en las Américas, desde el mero bautizo de Indias Occidentales a las tierras y las aguas que conectaban a Santo Domingo con las islas Filipinas, pasando por México y Perú. Me gusta pensar que lo que en general se entiende por posoccidentalismo, posorientalismo, poscolonialismo es una formación específica del proyecto, más amplio, de reflexión crítica sobre los legados coloniales.

Existen otras formaciones que no han tenido la misma suerte en el consumo, pero que son igualmente importantes para el proyecto de la crítica de los legados coloniales en vista a una continua descolonización intelectual que contribuya a cambio de proyectos educativos, a intervenciones intelectuales en la esfera pública y a contribuir en la orientación de las políticas culturales. Esas formaciones serían el posoccidentalismo, propuesto por Fernández Retamar en 1976 (Retamar, 1976), posorientalismo, que tuvo su primera formulación en Edward Said (Said, 1978; Mignolo, en prensa). El primero, vertido en castellano, pasó poco menos que desapercibido en la discusión intelectual internacional. El segundo, publicado en inglés y abarcando un espectro amplio de legados coloniales, desde la India al Medio Oriente, tuvo una enorme repercusión, aunque quedó marginado de la formación denominada poscolonial, quizás porque el legado 
colonial del Medio Oriente es más complejo que el de India, no tiene el inglés un lugar prioritario como en India, y para lidiar con esos legados coloniales es necesario remontarse más atrás del colonialismo inglés, en el momento crítico de las tensiones y los conflictos entre las tres religiones del libro (Cristianos, Judíos e Islámicos), al momento en que el imperialismo hispánico separa al Islam y lo sitúa en la exterioridad del imperio, y margina a los Judíos, como el "otro" interior (Shohat, 1993).

Por cierto que cuando hablo de legados coloniales ho estoy abogando por un esencialismo o autenticidad geográfica. Estoy mejor marcando las configuraciones geohistóricas tal como han sido construidas por los sucesivos diseños imperiales, no sólo hacia las áreas colonizadas sino también en la relación conflictiva con otros imperios (e.g., España con el Islam; Inglaterra con España y Francia; Francia en conflicto con Estados Unidos en América Latina, etc.). Pensar en la organicidad entre lengua, cultura y territorio sería sólo posible dentro de la epistemología colonial/moderna, que separó el espacio del tiempo, fijó las culturas a territorios y las localizó atrás en el tiempo de la ascendente historia universal de la cual la cultura europea (también fija a un territorio) era el punto de llegada y de guía para el futuro. Así la cristianización, la misión civilizadora ocuparon los proyectos coloniales europeos hasta 1945, y el desarrollismo/ modernización consumista los remplazó como proyecto colonial después de 1945, liderado por Estados Unidos. Este es, pues, el problema sobre el que aquí voy a reflexionar. Los legados coloniales son variados. No sólo porque hubo muchos colonialismos e imperialismos superpuestos a ellos ${ }^{1}$, sino porque las localizaciones geográficas donde operan los distintos colonialismos varían.

Ahora bien, una vez que se consideran los legados coloniales (es decir, el imaginario colonial en el presente que mantiene contradicciones no resueltas en el pasado), nos damos cuenta que tales legados coloniales son un espacio de acumulación de furia que no se articula teóricamente, porque la teoría ha estado siempre del lado civilizador de los legados coloniales, nunca del lado de la fuerza dividida entre la civilización y la barbarie. Esa epistemología fronteriza, que quedó siempre reprimida como lo impuro y lo mixto, frente a teorías que defendían la unidad del idioma, la pureza de sangre y de la razón (no contaminada por las emociones), comienza a surgir hoy no sólo con la riqueza de la civilización incorporada a la barbarie, sino también con la furia del engaño histórico convertido en toma de conciencia. Esta toma de conciencia se facilita cada día más por lo que se conoce como la última etapa de la globalización, en la cual comienza a hacerse problemático concebir - como se lo había hecho - la expansión colonial e

\footnotetext{
1. Entiendo por "colonialismo" la etapa de expansión colonial desde 1500 hasta 1945, que incluye España, Portugal, Holanda, Inglaterra, Francia y Alemania, principalmente; y por "imperialismo" la etapa que comienza despues de la segunda guerra mundial con la hegemonía de los Estados Unidos. Básicamente, el colonialismo es territorial, opera por control de territorios, en tanto que el imperialismo opera transnacionalmente y por el tecnoglobalismo de la imagen televisiva y del internet.
} 
imperial como expansión occidental; una expansión que era no sólo económica o religiosa, sino también educativa e intelectual. Tanto se exportaban educadores, como tecnología como teorías a las distintas partes del planeta. Pero los educadores, la tecnología y las teorías y el conocimiento fuerte y sostenible, se producían en Europa, primero y en Estados Unidos después. El conocimiento producido en las colonias o en las áreas reguladas por los diseños imperiales, si era interesante lo era como objeto de estudio que permitía comprender formas locales de vida, pero que no se consideraba parte del saber universal, producido por la humanidad.

La última etapa de la globalización está haciendo posible una transformación radical de la epistemología al llamar la atención entre espacios geográficos y localizaciones epistemológicas. No, claro está, porque haya nada telúrico en el espacio geográfico que llama a un determinado tipo de reflexión (y que le permitía a Pablo Neruda imaginar que hendía la mano en lo más genital de lo terrestre), sino porque los espacios geográficos son espacios configurados por historias coloniales. Son no sólo historias locales, sino localizadas. Y a menos que se siga pensando, con René Descartes (Toulmin, 1990:545; Dussel, 1993, 1995), que hay un sujeto universal y des-incorporado del conocimiento que piensa en ningún lugar y que lo que piensa vale tanto para los legados coloniales en Bolivia como en India, no nos queda otra posibilidad que in-corporar la producción de conocimientos que fue des-incorporada por la gestación del concepto moderno de razón y de conocimiento.

Finalmente, una nota sobre la relación entre estudios culturales, crítica a la razón imperial/occidental/colonial y formación de la razón post/imperial/occidental/colonial. Hay dos momentos en la formación de los estudios culturales, en Birminghan, que me interesa subrayar. El momento fundador que ha trazado Raymond Williams y el momento del giro de la crítica a la razón occidental que inserta Stuart Hall. Un tercer momento sería el del ingreso de los estudios culturales en U.S., para los cuales un rasgo distintivo sería la atención que presta a la cultura de masas, a las formas de consumo, formas que corresponden a lo que Hall mismo llama la nueva etapa de globalización liderada por U.S., y no ya por Inglaterra (Hall, 1990a:27). En este amplio terreno donde se mueven los estudios culturales, mi interés particular parte de lo que llamaré "el giro Stuart Hall". Ese giro se estructura con más claridad hacia 1990 (Hall 1990), aunque podrían encontrarse momentos anteriores a esa fecha para quien le interese una arqueología del pensamiento de Hall (Morley and Chen, 1996). Los 90 son el momento en que en el pensamiento de Hall confluyen la emergencia de los estudios culturales y la crisis de las ciencias humanas (ciencias sociales y humanidades), la crítica a la razón occidental/imperial y la reflexión crítica a la globalización como un nuevo momento donde la razón occidental/imperial se rearticula a sí misma por medio de un nuevo giro de la economía capitalista que transgrede las fronteras que la razón occidental/imperial había trazado para justificar su propia expansión (Hall, 1991a, 1991b). 
Ese momento crucial, el del "giro Stuart Hall", es el momento en que el análisis de las dos formas actuales de globalización (aquella que está ligada a estados nacionales imperiales, U.S. y aquella que transciende las fronteras nacionales, es decir, la globalización de un capitalismo sin arraigo territorial y nacional), crea las condiciones para - al decir de Hall - la emergencia de lo local: el sujeto local, de las márgenes, comienza a contar sus propias historias, a construir una memoria que había sido, o bien ignorada, o bien contada desde la razón occidental/imperial. La inversión epistemológica fundamental que marca la inserción de lo post-occidental-colonial imperial en los estudios culturales es que las historias comienzan a contarse desde abajo hacia arriba, en vez de desde arriba hacia abajo (Hall, 1990a:35). Esta imagen invierte la imagen que los viajeros europeos solían usar para presentar su posesión epistemológica: el viajero generalmente relata el momento en que se sube a un promontorio, a un lugar elevado, desde arriba, donde la mirada absorbe y conoce (debo esta observación a Mary-Louise Pratt, Bogotá, Marzo 1996); el momento desde arriba, en el cual el montar es al mismo tiempo poseer, aunque en la desexualización epistemológica de la modernidad se haya ocultado la relación entre epistemología y sexualidad. El momento posterior a la segunda guerra mundial, es el momento en que la epistemología del promontorio comienza a desmoronarse y, al decir de Hall, aparece el momento en "que lo no hablado descubre que tiene una historia que se puede hablar, que hay otros lenguajes que los lenguajes del amo (Hall, 1990a:35)." La cuestión no es por cierto que los subalternos no puedẹn hablar, sino que al tomar conciencia de que los subalternos no pueden hablar, es necesariamente hablar constantemente para inscrustar la voz en la espesura hegemónica y crear las necesarias fisuras mediante la insersion de lo local, desde abajo, en lo global, desde arriba del promontorio.

\section{II}

La ecuación entre el lugar geográfico y la teoría (al igual que la producción tecnológica de conocimientos), está relacionada con la ecuación moderna entre tiempo y teoría (y producción tecnológica de conocimientos). La etapa actual de globalización está reconvirtiendo la prioridad que la modernidad puso en una progresión lineal y temporal de la historia universal, en la simultaneidad espacial de las historias locales. Si podemos distinguir entre historia universal (como progresión de la humanidad hacia la etapa mas alta de la civilización, que Hegel ejemplificaba con el corazón de Europa: Alemania, Inglaterra y Francia), y la historia mundial como la multiplicidad de historias locales, entonces podemos afirmar que hoy - mediante el internet y la comunicación televisiva vía satélite - la historia universal no es sólo una que se cuenta desde determinados espacios geográficos y epistemológicos (por ejemplo, la lecciones de historia universal de Hegel), sino que las historias mundiales son muchas, precisamente porque 
sólo pueden contarse desde una encarnación local y no desde un sujeto desencarnado que observa la historia universal desde el lugar asignado a diós, fuera de la historia. Historias locales interconectadas, cuya interconección la historiografía colonial tendió a ocultar.

Quizás fue recién en 1974, con la publicación de Immanuel Wallerstein sobre el sistema mundo (Wallerstein, 1974), que la posibilidad de historias locales en los lugares de intersección de las expansiones coloniales e imperiales comenzó a pensarse sistemáticamente. La distinción de centro y periferias, introducida por Wallerstein, ayudó a tomar conciencia de que las periferias son también ellas centro. No es por casualidad, para ponerlo de manera concreta, que Heidegger haya reflexionado sobre el ser y el tiempo, y no el ser y el espacio; que Proust haya escrito a la búsqueda del tiempo perdido, y no del espacio perdido; que Bergson haya reflexionado sobre la memoria y no sobre la localización. En fin, la concepción lineal del tiempo en la modernidad, ligado a la historia universal fue un instrumento de dominación colonial que redujo el resto del planeta a una anterioridad histórica en relación a Europa (Fabian, 1982; Mignolo, 1995) primero y a Estados Unidos después. El legado de los antiguos colonialismos justificó la ideología del desarrollo y del subdesarrollo, con todas las consecuencias que tuvo en la producción del conocimiento en América Latina: desde las teorías del desarrollo de la CEPAL hasta la teoría de la dependencia, que se opuso a ella, pasando por un marxismo dogmático que no entendió la teoría de la dependencia debido a la auto-colonización de sus pensadores, que prefirieron convertir las observaciones de Marx sobre el siglo XIX en Europa en dogmas para entender América Latina en el XX, en lugar de comenzar a pensar a partir de las historias locales (Marini, 1994).

La reflexión sobre espacios geográficos y localizaciones epistemológicas es posible y es promovida por las nuevas formas de conocimiento que se están produciendo en las zonas de legados coloniales, en el conflicto fronterizo entre historias locales y diseños globales, desde América a Africa del Sur, desde América hasta Africa del Norte; desde el Pacífico en las Américas hasta el Pacífico del sur de Asia y Oceanía. En esta reflexión no se trata sólo de recoger datos, y de contar el cuento de lo que pasó y de lo que pasa. Se trata, más bien, de entender la fuerza de las epistemologías fronterizas, de aquellas formas de conocimiento que operan ENTRE los legados metropolitanos del colonialismo (diseños globales) y los legados de las zonas colonizadas (historias locales). Se trata de pensar a partir de esta situación, una nueva situación histórica que necesita de una nueva epistemología, así como lo comprendió René Descartes hacia 1630, cuando asomaba una nueva etapa en los diseños imperiales a la cual su reflexión metodológica contribuyó a implementar. La epistemología fronteriza que se anuncia para el futuro es al mismo tiempo, el desplazamiento de la epistemología de fundación cartesiana, así cómo ésta en su momento anunció el 
desplazamiento de una epistemología que contribuía a los diseños globales del cristianismo y del mercantilismo esclavista. Voy a detenerme en algunos casos para darle algún anclaje a las reflexiones que preceden.

\section{III}

Hacia finales de los años sesenta, el "antropologador" (como se llamaba a sí mismo) brasileño Darcy Ribeiro publicó dos libros fundamentales, "O processo civilizatório" (1968) y "Las Américas y la civilización" (1968). Ribeiro buscaba una explicación al "desarrollo desigual de los pueblos," enmarcándolo en el vocabulario que las teorías del desarrollo y la modernización habían impuesto alrededor de 1960 (Cardoso, 1972; Escobar, 1995) y la encontró - por su formación antropológica - pensando el proceso civilizatorio como una variedad ramificada de procesos locales que se contraponía - en silencio - a la visión dominante impuesta por el sociólogo alemán Norbert Elías en su "El proceso civilizatorio" (1937). Este, en su magistral manejo de la reglas disciplinarias (un sociólogo con todas las de la ley, y no un antropologador fuera de la ley), consideraba como tal el proceso de la civilización europea a partir de 1500 hasta el momento que se escribió el libro, entre la primera y la segunda guerra mundial.

Ribeiro enmarca los procesos de encuentros conflictivos y de poder a partir de 1500 como formas de sometimiento económico y cultural: Sometidos a los mismos procedimientos de deculturación y a idénticos sistemas productivos que se organizaban de acuerdo con formas estereotipadas de dominio, todos los pueblos alcanzados se empobrecieron desde el punto de vista cultural. Cayeron así en condiciones de extrema miseria y deshumanización, que vendría ser desde entonces el denominador común del hombre extra-europeo $(1969,60)$. De esta manera, Ribeiro podía vincular en el emprobrecimiento cultural al empobrecimiento epistemológico: Así como Europa llevó a los pueblos abarcados por su red de dominación sus variadas técnicas e inventos (como los métodos para extraer oro o para cultivar la caña de azucar, sus ferrocarriles y telégrafos), también introdujo en ellos su carta de conceptos, preconceptos e idiosincracias referidos a sí mismos y al resto del mundo, incluidos los correspondientes a los pueblos coloniales. Estos, privados de las riquezas por siglos acumuladas del fruto de su trabajo bajo el régimen colonial sufrieron, además, la degradación de asumir como imagen propia lo que no era más que un reflejo de la visión europea del mundo que los consideraba racialmente inferiores por ser negros, indígenas, o mestizos (1969:62).

La liberación epistemológica puesta en marcha por la epistemología fronteriza consiste, precisamente, en las consecuencias de desarticular la creencia en una imagen propia que no era más que un reflejo de la manera en que el discurso colonial producía agentes subalternos. Esos agentes subalternos, asignados como bárbaros o primitivos y 
cuya asignación aceptaban, están transformándose en agentes que parten de la subalternidad para producir una transformación epistemológica que incida en las políticas culturales. El espacio entre la formación y transformación de conceptos es a veces difícil de transitar. Pero intentemos, al menos, comprender cuáles son las posibilidades. Tomemos un aspecto de la recepción de "O processo civilizatorio" y las consecuencias que pueden derivarse de la comparación con el estudio de Elías. En la introducción a la versión inglesa del libro, la antropóloga estadounidense Betty J. Meggers, destacaba que las propuestas de Darcy Ribeiro merecían atención, entre otras razones, porque en Estados Unidos se heredó la tradición de la civilización occidental europea (palabras empleadas por Meggers), "consideradas por nosotros como una corriente principal o central de la evolución humana" (1978:20), que es precisamente la perspectiva desde la cual Norbert Elias escribió su libro "El proceso civilizatorio". Como consecuencia de esa creencia, continuaba Meggers, medimos a todos los otros pueblos con nuestra vara, los consideramos carentes de algo y consideramos que el progreso es hacer a los otros pueblos semejantes a nosotros.

Ribeiro, para Meggers, no es un producto de "nuestra tradición política y académica" (Meggers, 1978: 20). Es un ciudadano, decía Meggers hacia principios de 1970, un ciudadano del Tercer Mundo y como tal "encara el desarrollo cultural con un prisma distinto y percibe matices que para nosotros permanecen encubiertos." (Meggers, 1978:20). Finalmente, Meggers notaba que no se trataba de autocriticar la academia americana para dar a Ribeiro una imparcialidad en la comprensión del proceso civilizatorio que la academia estadounidense había perdido. Lo que en última instancia destacaba Meggers era la legitimidad de diferentes perspectivas para entender el proceso civilizatorio, articuladas por aquellos que lo viven y lo perciben en distintas historias y epistemologías locales. Se podrían emplear las observaciones de Meggers para comprender la desconfianza que intelectuales y científicos sociales latinoamericanos y latinoamericanistas de otros países tienen hacia el trabajo de Darcy Ribeiro. Se podría decir, hay una desconfianza de la civilización disciplinada del conocimiento que se resiste a aceptar la brillantez de la teorización bárbara que incorpora la civilización. Meggers, con sus observaciones, nos brinda la oportunidad de reconocer en Elias la disciplina civilizada que reflexiona sobre el proceso civilizatorio del cual la misma sociología histórica es parte, y la disciplina indisciplinada que trata de escapar al control civilizador de las ciencias sociales y de su complicidad con los diseños imperiales.

La importancia de lo que señala Meggers se comprenderá mejor si recordamos los prejuicios celebratorios con que Heinz Rudolf Sonntag se refiere a Ribeiro en el epílogo de la edición alemana del mismo libro. Con gran reconocimiento hacia Ribeiro, Sonntag declara que "el proceso civilizatorio" es una teoría del Tercer Mundo para el Tercer Mundo (1978:216). En última instancia tiene razón Sonntag y, siguiendo su lógica, podemos decir que el materialismo dialéctico marxista o el psicoanálisis freudiano son teorías del Primer Mundo para el Primer Mundo. Pero entonces, ¿cómo es que las 
teorías del Tercer Mundo son sólo locales mientras que las teorías producidas en Europa o en Estados Unidos son exportables y de valor global? Meggers, en cambio, había entendido otra cosa: que si no hay diálogo entre conocimientos locales, hay exportación y colonización de unos conocimientos sobre otros.

La exportación de conocimientos, al igual que la exportación de formas de estado, es parte del proceso de autocolonización que se describe en la cita de Ribeiro, más arriba. Tanto el proyecto de exportación que caracterizó la misión civilizadora, como el proyecto de importación de las burguesías locales, fueron resultado del pacto "de asumir como imagen propia lo que no era más que un reflejo de la visión europea del mundo". En la medida en que el mundo, desde 1500, fue distribuido entre occidentales y orientales, entre cristianos y salvajes o caníbales, entre primitivos o bárbaros y civilizados, entre habitantes del Primer o del Tercer Mundo, el espacio geográfico fue marcado también por localizaciones epistemológicas, y los conocimientos comenzaron a circular y a exportarse o a importarse, como el oro en el siglo XVI o la coca cola en el siglo XX. Pero veamos algunos casos específicos del complejo exportación/importación de conocimientos y en los procesos de colonización/autocolonización.

\section{IV}

Tomemos un caso de la historiografía hindú conocida como "Estudios Subalternos". Dipesh Chakrabarty (1992), se planteó el problema de la localización de la disciplina historiográfica en los estudios subalternos. El planteamiento fue suscitado por una serie de discusiones previas, a nivel internacional, como parte de la discusión promovida por el impacto de los estudios subalternos. En 1990, Gyan Prakash (historiador de India, afiliado a la universidad de Princeton), publicó un artículo titulado "Writing Post-Orientalist Histories of the Third World: Perspectives from Indian Historiography" (Prakash, 1990), en el cual se preguntaba si era posible escribir historias del Tercer Mundo y si lo era cómo deberían ser escritas. Sus preguntas dejaban planteada otra: ¿es posible escribir historias del Tercer Mundo desde la perspectiva del Tercer Mundo? $\mathrm{O}$, por el contrario, las historias del Tercer Mundo sólo pueden escribirse desde el Primer Mundo, es decir, desde las pautas y principios de la historiografía como práctica disciplinaria, ejercida en la producción de conocimientos SOBRE (pero no DESDE) el Tercer Mundo? Si la historiografía en Occidente fue parte y cómplice de la producción del "orientalismo" (conocimiento SOBRE el oriente) ¿será posible escribir historias "posorientalistas" del Tercer Mundo, transformando así el objeto de estudio para la disciplina historiográfica en sujeto y agencia política que reune en el conocimiento el SOBRE con el DESDE, lo conocido con la localización del conocer?

Una de las respuestas programáticas ofrecida por Prakash comienza por una reconsideración de las contribuciones de los estudios subalternos (Guha, 1988) y de las propuestas de Said (Said, 1978). Guha identificó tres tipos de historiografía de India 
que sucedieron a la historiografía del colonialismo británico: la del nacionalismo hindú bajo control del imperialismo británico, durante el siglo XIX; la del nacionalismo posterior a la independencia de 1947; y los estudios subalternos iniciados hacia 1980. Todas ellas son manifestaciones de cómo en el Tercer Mundo los intelectuales escriben la historia. Sin embargo, el momento clave para responder a la pregunta formulada por Prakash, es la introducción por parte de Guha del concepto de "subalternidad" en la historiografía. La subalternidad se convierte así en un juego de fuerzas y relaciones sociales de dominación que incluye y supera el concepto marxista de clase. La subalternidad es un efecto de las relaciones de poder que se expresa a través de una variedad de medios: lingüísticos, sociales, económicos y culturales.

La noción de subalternidad, introducida en la densidad de la experiencia colonial en India, adquiere una nueva dimensión en relación al concepto introducido por Gramsci en Europa y en la experiencia italiana de la lucha de clases. Sin embargo, los estudios subalternos han permitido corregir las limitaciones modernas del concepto de "cultura" en Gramsci y sus implicaciones para el concepto de "subalternidad" en la medida en que para Gramsci era pensable todavía el proyecto de incorporar la cultura popular a una "mentalidad moderna" (Rabasa, en prensa). La experiencia colonial permitió, en realidad, corregir los límites con los que se encuentra Gramsci, al incluir y superar las relaciones de clases y las correspondiente distinción entre Cultura y cultura popular posible de pensar en regiones que fueron agentes pero no pacientes de los procesos de colonización. Desde India, en cambio, las cosas se ven, aparentemente, de manera distinta. La fuerza de las empresas intelectuales desde el "Tercer Mundo" es la que ofrece simplemente la doble experiencia de manejarse al mismo tiempo en la epistemología de la modernidad occidental y en la diferencia de las epistemologías subalternizadas por la modernidad.

Esta epistemología fronteriza, que incorpora la civilización a la barbarie a la vez que niega el concepto colonial hegemónico de civilización, es la que encuentro implícita en Darcy Ribeiro. Y es precisamente desde esta epistemología fronteriza que el dilema planteado por Chakrabarty tiene una respuesta epistemológica y política: si no es posible hacer historia del Tercer Mundo desde el Tercer Mundo porque la historia es una disciplina del Primer Mundo, es necesario no confundir la construcción de la memoria con su disciplinamiento historiográfico. El dilema de Chakrabarty es el de la historiografía que, por un lado, conserva un determinado concepto de rigor académico y, por otro, es un remedo de formas coloniales de domesticar el pasado.

Antes de continuar el argumento es útil recordar aquí una interesante superposición de categorías geo-históricas: "orientalismo," después del análisis de Said, es una construcción Europea del oriente; Tercer Mundo en cambio, es el equivalente geocultural de orientalismo en el momento en que Estados Unidos reemplaza a Francia, Inglaterra y Alemania en la hegemonía mundial y rearticula las áreas geográficas en relación al conocimiento. Pues bien, este tipo de problemas es el que aborda Chakrabartý. 
Trasladado al ámbito disciplinario este asunto podría formularse así: los estudios subalternos, ¿son una rama de la historiografía que estudia el papel de los subalternos en la historia de India, o es una historiografía subalterna, como disciplina, dependiente de la historiografía hegemónica institucionalizada en la modernidad occidental (del atlántico norte)? La respuesta de Chakrabarty es que este último es sin duda el caso.

Puesto de otra manera: la historiografía es una práctica disciplinaria exportable, como la tecnología para la irrigación de los campos, que puede adaptarse - otra cosa son las consecuencias a distintas historias locales (o partes del planeta). No obstante, la subalternidad disciplinaria es la que le da su fuerza debido, precisamente, a la posibilidad que tiene de postularse como la irrupción de la brillantez bárbara, frente al control constipatorio de las disciplinas y los agentes disciplinadores que, muchas veces, confunde rigor académico con autorrepresión. Lo cual significa que se está frente a una opción: la de sujetarse al control de la seriedad disciplinaria o la de rebelarse frente a las condiciones de conocimiento disciplinado que emplea el concepto de razón académica para gobernar lo que comienza convertirse en ingobernable, de la misma manera que el párroco invoca a Dios, en vez de la Razón, para sujetar la conducta de los pecadores. La fuerza ingobernable de la razón pos-oriental (no como antítesis del orientalismo, sino como su superación), una razón que parte de, incorpora y supera la razón moderna y corroe sus mecanismos de sujeción. En América Latina es posible plantear un problema semejante con respecto al occidentalismo y de la razón pos-occidental, teniendo en cuenta claro que el occidentalismo, por un lado, no es el reverso del orientalismo sino su condición de posibilidad y, por otro, que América Latina se construye históricamente no como Oriente sino como el margen de Occidente. Pero volvamos a la India y al orientalismo.

Quizás este mismo problema se pueda hacer más claro con el caso de la importación de Sigmund Freud, a Calcuta, a principios del siglo XX, estudiado por Ashis Nandy (1995). La historia, según la cuenta Nandy, es la de un tal Girindrasekhar Bose (nacido en 1886, en una familia de Bengala occidental), quien tradujo a Freud por los años 20 y creó la primera sociedad psicoanalítica no-occidental. Su padre trabajó para un amo inglés cuando joven, pero cuando Girindrasekhar nació era marajá, y se conformaba a lo que la sociedad urbana de Bengala denominaba un "gentleman." De tal modo que cuando Girindrasekhar alcanzó su edad adulta, la suya era una familia reconocida, respetada y comprometida con el estudio. Por ésta y otras razones, Girindrasekhar tenía un buen conocimiento del hindú, del sánscrito y, por cierto, del inglés. Finalmente, la familia se mudó a Calcuta lo que le permitió a Girindrasekhar entrar en la escuela de medicina y convertir la casa de su familia en el centro del psicoanálisis en India. 
Desarrolló también, desde temprano, un interés particular en la magia y el hipnotismo. No sólo eso, sino que llegó a publicar artículos en cuestiones de magia y empleó el hipnotismo para curar a pacientes con casos de insomnio. Estaba por los cuarenta cuando se estableció como doctor, hacia 1926-27, y se concentró en caso de desórdenes mentales.

Lo anterior, aunque una biografía simplificada, es importante para darse cuenta del contexto en el cual, quizás hacia 1918, Bose comenzó a leer a Freud en traducciones al inglés y se sorprendió de la similitud entre concepciones que él mismo había desarrollado a partir de sus lecturas y experiencias, por un lado, y las teorías de Freud, por otro. Cuando su tesis para el nuevo departamento de psicología (inaugurado en Calcuta en 1915) fue publicada (alrededor de 1920), Girindrasekhar le envió una copia a Freud. A partir de ahí inició una correspondencia con Freud que duró casi dos décadas y al poco tiempo de fundada en Inglaterra la British Psychoanalytic Society, Girindrasekhar fundó la Indian Psychoanalytic Society in Calcuta, en su propia residencia.

He contado esta breve historia, resumiendo a Nandy, para hacer más intelegibles los tres puntos siguientes:

1) El reconocimiento en India del psicoanálisis ponía a Freud en un brete, puesto que el de Bose era el reconocimiento intelectual del mundo "primitivo" al cual el psicoanálisis, como otros discursos amarrados en la idea de procesos civilizatorios y de mundos, negaba. A las zonas bárbaras, donde la misión civilizadora tenía precisamente que cumplir su misión, no les correspondía teorizar porque si teorizaban la misión se quedaba sin misión. ¿Cómo podrían pues civilizar a gente que era ya civilizada? Bose indirectamente (y quizás inconscientemente!) mostró que las formas "civilizadas" de conocimiento tenían en el concepto de civilización la autolegitimación de su propia autoridad. Después de todo, el consumo del psicoanálisis en India era parte de la trayectoria de la "misión civilizadora" de los imperios de turno a principios del siglo XX.

2) Era también una paradoja que el psicoanálisis, castigado en Europa, fuera reconocido en las colonias del imperio inglés.

3) Finalmente, y como era de esperar, el psicoanálisis no tuvo arraigo en India, por entonces, puesto que ¿cómo una teoría que trata de resolver problemas que se le crean a la conciencia moderna de sujeto (Giddens, 1991), que se construye sobre los fundamentos de la Ilustración para lidiar con desórdenes mentales en la sociedad Europea de clases, a finales del XIX y del XX, podría tener arraigo en una sociedad de castas, con un fuerte anclaje en una ética basada en textos canónicos de India? Y esto es, en verdad, lo que Bosse trató de hacer: compaginar a Freud con palabras-claves de los códigos culturales milenarios en India. Es posible entender que el psicoanálisis se haya arraigado en Buenos Aires, una sociedad de inmigrantes europeos (Balan, 1991; Vezzetti, 1993), sin chamanes como en Venezuela o en los Andes.

El párrafo anterior permite introducir, con cierta naturalidad, la conferencia inaugural dictada por Jacques Derrida, en 1981 y en Paris, para el Encuentro Francés-Latino 
Americano de la Sociedad Internacional de Psicoanálisis ${ }^{2}$. Arriesgando sintetizar una conferencia de Derrida, se puede decir que toda ella gira en torno a dos temas superpuestos: una es la localización geográfica del psicoanálisis; la otra es la relación entre psicoanálisis y derechos humanos, lo que pone sobre la mesa la relación entre psicoanálisis y violencia en las áreas geográficas donde esto ocurre. Y como este encuentro convocaba a psicoanalistas latinoamericanos y era 1981, cuando la violencia y la violación de los derechos humanos en Argentina tocaba a distintos lugares del mundo, el cuestionamiento de la relación psicoanálisis violencia política se imponía.

Primero, pues, la relación entre psicoanálisis y geografía, o geopsicoanálisis como el mismo Derrida propone. Derrida parte de las declaraciones impresas por la propia Sociedad Internacional de Psicoanálisis, que constituyen su descripción y su fundación. Una de esas declaraciones ubica al psicoanálisis geográficamente: Las principales áreas geográficas de la Asociación se definen en este momento (Jerusalem, 1977) como América al norte de la frontera entre México y Estados Unidos; y toda la América al sur de esa frontera; y finalmente el resto del mundo (p.141). Quién no ha leído el artículo supone bien al pensar que Derrida hace una fiesta con la expresión "el resto del mundo," pero no hay lugar aquí para comentarla. Lo que sí interesa no es sólo la distribución geográfica establecida por la Asociación, sino también dónde y dónde no el psicoanálisis asienta sus reales ("en este momento."). Sabemos, por ejemplo, que en 1977 en Argentina sí pero en México (y en la mayor parte de América Latina) no. En 1981 quizás puedan agregarse México (Pasternak y Braustein se exilaron hacia mediados de los 70), y quizás Brazil y Chile. Pero seguramente no en Bolivia, Perú, Ecuador, Colombia, América Central, y todas las islas del Caribe, incluidas las francesas y a pesar de la obra de Fanon. Y sabemos también que en Estados Unidos el psicoanálisis tiene un radio limitado de acción tanto geográfica como epistemológicamente, donde el desarrollo teórico y crítico, en las humanidades, supera el de la práctica psicoanalítica.

Pero de todas maneras, y dejando de lado el balance empírico, lo cierto y lo importante es que las Américas, al sur y al norte del Río Grande son un lugar propicio para la instalación del psicoanálisis fuera de Europa. En cambio, el "resto del mundo" (Asia y Africa, para simplicar) no lo es, como bien lo comprobó Bosse en Calcuta en la primera mitad del siglo XX. Las Américas, contrario a Asia y a India fueron, desde 1500, el lugar de la extensión del Oeste Europeo: las Américas no se configuraron como América, sino como las Indias Occidentales y cuándo América comenzó a reemplazar el nombre originario - cuando España caía, los imperios al norte de los Pirineos subían, y América del Norte emergía - bien pronto se nombró todo un hemisferio, el hemisferio occidental. Asia y parte de Africa, en cambio, pasaron a constituir el

2. Estoy agradecido a Horacio Legras por haberme indicado este artículo de Derrida, en conección con un seminario donde habíamos discutido cuestiones de localizaciones geográficas y epistemológicas. 
hemisferio oriental como fundación de lo que Edward Said (Said, 1978), describió y explicó como "Orientalismo."

Resulta sin duda interesante que Derrida, en el artículo citado, haga referencia a Frantz Fanon, en un párrafo donde él mismo (Derrida), se sitúa geo-culturalmente. Ubicándose, primero, con respecto al psicoanálisis del cual se considera extranjero (ni es analista por entrenamiento ni tampoco se analiza), agrega que su extranjería es doble: dada la característica geo-cultural del congreso en el cual presentó la conferencia inaugural (Congreso Francés-Latino Americano), señaló que tampoco es americano (ni del norte ni del sur), sino nacido en Africa y subrayó : "I guarantee you that I retain something of that heritage.” La razón por la cual Derrida mencionó este hecho era para sostener que no hay psicoanálisis en Africa, como tampoco en Asia o en los mares del Sur. Al mencionar a Fanon, Derrida lo hizo también para destacar cuan poco usual era, en los sesenta, formular la práctica psicoanalítica en relación a la dimensión política de su propia práctica. Merece la pena citar a Derrida en este punto:

The laws, the deontology, the ethics, of psychoanalisis, as laid down or simply taken for granted by the colonial societies or by the international psychanalytic establishment were supposed to regulate practice and govern relations with state authorities on the one hand and medical authorities on the other; I say this merely in order to provide a well known and painful point of reference: Fanon; own position as a model beyond the reach of all discussion. The political geography of the world has changed since that time, and intercontinental balances of power have been subject to much turbulence; this can hardly have failed, it seems to me, to have had an impact on the political geography of psychoanalisis. (Derrida, 1995:144-145)

Antes de abordar el segundo punto discutido en la conferencia de Derrida, necesito hacer un desvío y aprovechar de las observaciones del párrafo anterior para insertar la relación entre distribución de áreas geográficas y exportación de conocimientos. El psicoanálisis (como la religión, las reglas del juego económico y los productos de consumo), además de otras prácticas disciplinarias (académicas o no, puesto que el psicoanálisis no es una práctica académica), era y todavía es exportable ${ }^{3}$. Quizás no convenga decir del centro a la periferia, pero sí se podría decir de su lugar de gestación

${ }^{3} \mathrm{La}$ creciente transnacionalización que hace que las mercancías fabricadas en distintas partes del mundo pidan un nuevo modelo que trascienda la relación importación/exportación basada en los Estados nacionales, no deberían ocultar la vigencia todavía del otro modelo. En Japón, por ejemplo, la situación es distinta a la de América Latina y el modelo importación/exportación es menos sostenible. En América Latina, en cambio, el modelo tiene vigencia por la tensión existente entre los nacionalismos y latinoamericanismos de tipo fundamentalistas, y la tendencia al comospolitanismo y la globalización (García Canclini, 1995). 
en la Europa imperial de principios del XIX y del XX, hacia zonas donde las pautas de vida y de conducta, de la economía y de la ley, se habían extendido. En última instancia, el psicoanálisis - como lo señalé más arriba - que se gesta y se empieza a exportar en el contexto de la "mission civilizatrice", contribuye a extender la civilización y controlar la barbarie. En realidad se trata de ambas, de exportación y de importación.

La exportación del psiconálisis ocurre en aquellos lugares, por ejemplo en Africa, donde el psicoanálisis es un puesto creado por la administración colonial y que como tal, y como lo dice Derrida, "where it has never taken off its European shoes." El otro caso es la importación, como el caso de Bose en India o como el psicoanálisis en Argentina. La diferencia entre India y Argentina es que en el primer caso, aunque fuera importación, lo fue en un régimen colonial. En el segundo, funcionó como mercadería importada por la intelectualidad de un país con poco más de cien años de lucha por la autonomía nacional, en un momento (del 1920 al 1930) en que es posible ser intelectualmente cosmopolita y en el que ya se ha asentado, en Buenos Aires, una masa de inmigrantes europeos. El psicoanálisis, en Argentina, es parte de un doble proceso de occidentalización: el de la inmigración para domesticar la barbarie (al menos en los planes oficiales), y el psicoanálisis para sujetar a la inmigración que llegada a domesticar la barbarie. En 1981 las cosas han cambiado y esa época apoya el segundo punto desarrollado por Derrida a raíz del Encuentro FrancésLatino Americano en el momento de la más radical violación de los derechos humanos en Argentina.

La pregunta latente en la discusión de Derrida es ¿qué relación hay entre la importación exitosa del psicoanálisis en una sociedad donde la violencia pública llega a niveles difícil de comprender? Por cierto que la ecuación no es de lógica histórica de tal manera que la violación de los derechos humanos presupondría el arraigo del psicoanálisis y viceversa. No obstante, la pregunta latente invoca otra, curiosamente ni siquiera sugerida por Derrida: ¿qué relación hay entre la lengua y la cultura en la que se gesta el psicoanálisis y la lengua y la cultura donde arraiga y florece el nazismo? De qué manera la inversión del proyecto de la Ilustración que se manifiesta en las dos guerras y en el nazismo, da lugar a formas de barbarie producidas por el propio proceso civilizador apoyado en los principios universales de la Ilustración, del cual el mismo psicoanálisis es un resultado y una necesidad? Pregunta inquietante, sin duda, y estrechamente ligada a la ecuación localización geográfica y producción de conocimientos.

Pero lo que más nos interesa aquí no es la producción sino la subalternización de conocimientos. Y bien, retomando la distribución de áreas establecida en la Constitución de la Asociación Internacional de Psicoanálisis, de lo que se trata en última instancia en la exportación importación de formas de conocimiento y de prácticas disciplinarias, es de la subalternización lo cual, en el área del conocimiento, supone el borroneo de las 
condiciones de emergencia de una práctica disciplinaria o de consumo y su adaptación o implantación en otras áreas geográficas con distintas memorias y necesidades.

Me pregunto si es equivalente instalar un McDonald en México, en una casa del siglo XIX cubierta por azulejos de talavera, e instalar (por exportación o importación) el psicoanálisis en Buenos Aires. Me lo pregunto porque me parece interesante reflexionar sobre el lugar de gestación y necesidades de prácticas de consumo y de prácticas disciplinarias. Me parece obvio que no hay necesidad de McDonalds en México como aparentemente tampoco hay necesidades del psicoanálisis en Bolivia; o al menos no la hay con la misma intensidad que la hay en Buenos Aires. No obstante, una vez instalado el McDonald en México y el psicoanálisis en Argentina, se implantan también pautas, aquellas inventadas e impuestas por Freud a partir de Viena y de Lacan a partir de París. Y sin duda, con todo el respeto por el genio de Freud y de Lacan, no había necesidad ni condiciones para que el mismo nivel de genialidad inventara y desarrollara el psicoanálisis en Argelia, en Bagdad o en la Argentina de principios de siglo.

Las condiciones históricas que producen a Freud y a Lacan, que inventan y desarrollan el psicoanálisis (que luego se exporta o se importa), son las condiciones históricas de los países imperiales y del corazón de Europa (Alemania-Viena, Francia, Inglaterra) en el momento álgido de la modernidad y el colonialismo. De aquí a la vinculación que subraya Derrida entre lugar de arraigo del psicoanálisis y violación de derechos humanos no hay más que un paso; y ese paso está relacionado con las vinculaciones entre psicoanálisis como mercadería exportable y la "mission civilizatrice" como estrategia de colonización.

El caso de Frantz Fanon merecía aquí un tratamiento especial. No sólo por la interpretación psicoanalítica del problema racial que desarrolla en Máscaras Blanca, Piel Negra (1952), sino también el trabajo psicoanalítico que desarrolló en la guerra de liberación de Argelia. Esta experiencia lo llevó a comprender no sólo la particular alienación de las personas en situaciones coloniales, sino también la enorme fractura que existe entre el psicoanálisis concebido y practicado en las lenguas y la cultura de Europa occidental, por un lado, y del mundo Arabe, por el otro. Quinientos años de conflicto entre el Islam y la Cristiandad, seguida por la Ilustración y la colonización francesa (1832), le dan a la práctica psicoanalítica en Argelia, un perfil muy distinto al de Calcuta y al de Buenos Aires.

\section{VI}

Mi último punto sirve al mismo tiempo de conclusión. Todos estamos familiarizados con la arqueología de las ciencias humanas analizada por Michel Foucault en "Les mots et les choses" (1966), de modo que no es necesario detenernos sobre ella. Lo que sí me interesa señalar son otros aspectos, no analizados por Foucault, que tienen que ver con la complicidad entre expansión imperial, culturas académicas y prácticas disciplinarias, académicas o no. Uno de ellos, rearticulado recientemente, aunque familiar 
para quienes se ocupan del colonialismo, es la ratio entre espacios geográficos, lenguas y epistemología (Mignolo: 1993, 1995, 1996).

Esta articulación se encuentra en el informe de la Comisión Gulbenkian, convocada para proponer una reorganización de las ciencias sociales después de la desarticulación del mundo socialista, de la pérdida de eficacia de una distribución del mundo en Primero, Segundo y Tercero y en sus consecuencias para la rearticulación de las ciencias humanas (empleo ciencias humanas, siguiendo la nomenclatura francesa, que reune ciencias sociales y humanidades, en la nomenclatura estadounidense). Esta comisión, efectivamente, reunió a un grupo que representaba en su mayoría de las ciencias humanas, pero también las naturales. Los miembros, tal como figura en la publicación del informe por Stanford University Press es la siguiente: Immanuel Wallerstein, Chair (E.U.); Galestous Juma (Kenya), Evelyn Fox Keller (E.U.), Jurgen Kocka (Alemania), Dominique Lecourt (Francia), V.Y. Mudimbe (Zaire), Kinhide Mushakoji (Japón), Ilya Prigogine (Belgica), Peter J. Taylor (Inglaterra) and Michel-Rolph Trouillot (Haiti). Una distribución, como se ve, geoepistemológica, puesto que cada uno de los representantes no sólo representan lugares sino disciplinas e invocan, indirectamente, la relación entre el espacio geográfico y la localización epistemológica.

Pues bien, una de las constataciones sobre las que se basa el informe es la de la relación entre emergencia de las ciencias sociales, en el siglo XIX, lenguas e imperios. El panorama sería a grandes rasgos el siguiente (Wallerstein, 1996): Al menos el 95 por ciento de la cultura académica (tanto en términos de investigadores como de becas y apoyos institucionales) entre 1850 a 1914, y probablemente hasta 1945, se originó en cinco países: Francia, Gran Gretaña, Alemania, Italia y los Estados Unidos. Si bien se pueden identificar, durante ese período, emergencias en otras partes, estas no llegan a igualar lo que ocurre en esos cinco países. Además, no sólo eso, sino que los investigadores se concentraron, en sus estudios, en esos países por considerarlos de mayor importancia para comprender el orden mundial.

Una de las consecuencias de esta situación es que, obviamente, esos cinco países no eran el mundo; peor aún, se tenía una vaga idea de que había un mundo fuera de esos cinco países. Pero una vez que se tomó conciencia de la existencia de ese "resto" se inventaron dos disciplinas que se ocuparan de él (y aquí me aparto ligeramente de Wallerstein): una, que se venía gestando desde el siglo XVIII, fue el estudio (comparado) de las civilizaciones, cuya repartición geoepistemológica fue "el resto" que se distinguía por sus religiones fuertes, religiones del libro en un caso (como el Islam), o religiones que se había extendido por anchas áreas geográficas (como el Budismo o el Hinduismo). Esto es, civilizaciones no-europeas distinguidas por la posesión de textos fundacionales, de carácter religioso y ético que, lamentablemente, no entraban en el paradigma secular de la modernidad y del progreso. Edward Said rebautizó el estudio de las civilizaciones y lo caracterizó ideológicamente como "Orientalismo". La segunda disciplina inventada para ocuparse del "resto" fue la antropología, que surgió en el 
momento en que los "salvajes" y "caníbales" de los siglos XVI y XVII fueron convertidos en "primitivos", y esta conversión acentuó la modernidad europea como la cúspide de un desarrollo temporal y lineal, y rebajó la contemporaneidad histórica del "resto" del mundo al situarla en un lugar "anterior" en el desarrollo universal de la humanidad, del cual Europa era el momento cúspide. Africa, al sur del mundo islámico, y ciertas zonas aisladas de Asia (espacios sin "civilización") se convirtieron en el lugar-objeto del conocimiento antropológico.

Pues bien, este panorama que giró en torno al control epistemológico de Europa hasta 1945, se adaptó a las nuevas circunstancias imperiales a partir de 1945 cuando el mundo se dividió en Primero, Segundo y Tercero (Pletsch, 1981; Mignolo, 1991, 1992, 1996, en prensa) y se inventó una tercera configuración disciplinaria que complementó a la antropología y que desplazó (en cuanto hegemonía académica) al estudio de las civilizaciones (aunque no necesariamente su dimensión ideológica, el "orientalismo"): estos nuevos invitados fueron los estudios de área (Berger, 1996). En esa distribución, el lugar de la producción de conocimiento se desplazó de Europa a Estados Unidos, en forma paralela al desplazamiento del poder económico y político. Las "áreas" (a diferencia de las "civilizaciones" y del "mundo primitivo") se definieron particularmente para el Tercer Mundo, como "áreas no-desarrolladas," "en vías de desarrollo" o "subdesarrolladas."

En esta nueva repartición entre Primer y Tercer Mundo, quedó el Segundo Mundo (económica, tecnológica y científicamente desarrollado pero víctima de impureza ideológica!). Y esto es muy importante, sin duda, puesto que ya no se trata de una impureza que toca al conocimiento disciplinario, sino a la ideología social: la fuerza que tuvieron los conceptos de pureza de sangre y de raza para la definición de la "otredad" comenzaron a adquirir una nueva dimensión. Aunque la cuestión racial persistió, apareció un nuevo virus que comenzó a infestar identidades de raza, clase social, género sexual y sexualidad: la subversión comunista como nuevo enemigo mundial. Aunque se trata de conocimiento común, es importante recalcarlo aquí puesto que nos devuelve a las observaciones de Derrida sobre la violación de derechos humanos y psicoanálisis en Argentina. La "guerra sucia" no estuvo separada de la producción y distribución de conocimientos después de 1945, ni a la doble tarea que se asigna Estados Unidos de controlar la expansión del comunismo y de contribuir al desarrollo y modernización del Tercer Mundo. Los estudios de áreas configuraron la dimensión epistemológica equivalente a la tárea de los misioneros, por un lado, y a la creación de la antropología en el siglo XIX, por el otro.

Pues bien, esta historia que estoy contando necesita de un nuevo personaje, que estuvo ausente de la escena aunque implicado en el escenario que acabo de describir. Ese personaje, con varios atuendos, podría nombrarse algo así como "principios de la epistemología moderna" (de Descartes, a Kant, a la Ilustración). Tal epistemología aceptaría, básicamente, que: 1) El mundo es objetivamente conocible y tal conocimiento 
puede generalizarse. No se aclara, sin embargo, dónde se produce el conocimiento del mundo y hacia dónde y bajo qué condiciones podría generalizarse; 2) El mundo objetivamente conocible genera conocimiento experto (economistas, antropólogos, ingenieros, técnicos en comunicación, agrónomos, psicoanalistas, arquitectos, etc.), se producen conocimientos que pueden exportarse o importarse (depende del lugar y de las condiciones bajo las que ocurre el viaje) como mercancía y ser aplicado a y en las historias locales y a las regiones (después de todo las regiones se constituyen para poder ser regidas); 3) La gestación del conocimiento experto presupone un espacio geohistórico en el que se construye una localización epistemológica desde donde se puede exportar conocimiento, de Europa a las dos Américas y al resto del mundo (recordando la división de la Asociación Internacional de Psicoanálisis). Freud se importa desde India como conocimiento experto, que entra en conflicto con el conocimiento no experto local, puesto que el conocimiento experto se asumía, como lo nota bien Wallerstein, en cinco países y en tres lenguas. El hindú y el sánscrito, desde la perspectiva europea que construye la noción de conocimiento basada en unos principios epistemológicos, son lenguas de cultura, no de conocimiento disciplinario.

Esta distinción hizo que el conocimiento local, en la distribución geo-epistemológica de la que hablamos, fuera sólo conocimiento local que puede ser objeto de estudio, pero que no es sostenible puesto que es de antemano rebajado como posibilidad de conocimiento. Paradójicamente, una de las consecuencias de la globalización económica actual de un capitalismo sin fronteras, está creando las condiciones para rearticular la epistemología moderna y parcializarla en el encuentro con el conocimiento local. El encuentro entre historias locales y diseños globales, va generando una epistemología fronteriza (precisamente entre lo global y lo local) para y desde la cual ya no sea ni posible ni aconsejable llevar, por ejemplo, sistemas de irrigación a India o a Bolivia sin anular la distinción entre el conocimiento experto del ingeniero que es totalmente inexperto en las formas de cultivo de India o Bolivia, y el conocimiento experto del campesino Hindú o Boliviano que es totalmente inexperto en materia de tecnología.

El punto de intersección entre historias locales y diseños globales da lugar a las epistemologías fronterizas como conocimiento crítico local (tanto en Europa como en América o Africa), debería restituir a los agentes locales el espacio para la producción de conocimiento suprimida por los mecanismos coloniales e imperiales de subalternización. Un argumento semejante se podría desarrollar con el psicoanálisis, la antropología, la sociología y, en fin, todo tipo de conocimiento académico y disciplinario que, originado en el Primer Mundo, ya no puede sostenerse como mera mercancía de exportación/ importación y necesita del diálogo entre expertos en las historias locales y expertos en los diseños globales. No se trata ya de desarrollo y subdesarrollo, sino de dos tipos distintos de "desarrollos" conducentes a transformaciones sociales para cuyos logros es necesario llegar al convencimiento de que la civilización, como lo subrayó Darcy Ribeiro, es un 
proceso que marcha en varias direcciones, y que las culturas académicas y tecnológicas no tienen la última palabra en materia de conocimiento.

La riqueza de la modernidad podrá potenciarse cuando se acepte que la modernidad es un monstruo de varias caras (occidentalismo, capitalismo, orientalismo, colonialismo) a la cual el planeta entero ha contribuido, aunque no todos hayan recibido los mismos beneficios y reconocimientos. El momento actual, de tendencia hacia los pos (occidentalismo, orientalismo, colonialismo, modernidad) puede ser tambien un momento en el que el poscapitalismo, que ya no depende de la unidireccionalidad imperial sino que está allí para quien lo agarre (por así decirlo), hace impensable la distinción entre occidente/oriente y, con ella, la desarticulación de todo el conjunto de categorías geoculturales que organizaron la distribución del poder en términos geoepistemológicos.

ESPACIOS GEOGRAFICOS Y LOCALIZACIONES EPISTEMOLOGICAS: LA RATIO ENTRE LA LOCALIZACIÓN GEOGRAFICA Y LA SUBALTERNIZACIÓN DE CONOCIMIENTOS

Resumen: Formulaciones epistemológicas deben ser relacionadas a espacios geográficos donde estean localizadas. Estas localizaciones epistemológicas ayudan a comprender los procesos de subalternización de conocimientos. El punto de intersección entre historias locales y diseños globales da lugar a las epistemologías fronterizas como conocimiento crítico local. Debería ser restituido a los agentes locales el espacio para la producción de conocimiento suprimida por los mecanismos coloniales e imperiales de subalternización.

Palabras-llave: Localización epistemológica, Epistemologia fronteriza, Teoria poscolonial

ESPAÇOS GEOGRÁFICOS E LOCALIZAÇÕES EPISTEMOLÓGICAS: A RATIO ENTRE A LOCALIZAÇÃO GEOGRÁFICA E A SUBALTERNIZAÇÃO DE CONHECIMENTOS

Resumo: Formulações epistemológicas devem ser relacionadas aos espaços geográficos em que estão localizadas. Estas localizações epistemológicas ajudam a compreender os processos de subalternização de conhecimentos. O ponto de interseção entre histórias locais e desenhos globais dá lugar a epistemologias fronteiriças como conhecimento crítico local. Deveria ser restituido aos agentes locais o espaço para a produção de conhecimento suprimida pelos mecanismos coloniais e imperiais de subalternização.

Palavras-chave: Localização epistemológica, Epistemologia fronteiriça, Teoria Pós-colonial

\section{BIBLIOGRAFIA}

BALÁN, J. 1991. Cuéntame tu vida: Una biografía colectiva del psicoanálisis argentino. Buenos Aires: Planeta.

CHAKRABART, D. 1992. Poscoloniality and the Artifice of History: Who Speaks for 
"Indian" Pasts? Representations 37, p. 1-26.

DERRIDA, J. 1995. Geosychoanalysis:... 'And the Rest of the World.'. New Formations 26, pp. 141-162.

ELIAS, N. 1989 (1937). El proceso de la civilización. Investigaciones sociogenéticas y psicogenéticas. México: Fondo de Cultura Económica.

FANON, F. 1952. White Masks, Black Skin. N. York: Grove Press.

FERNÁNDEZ RETAMAR, R. 1976. Nuestra América y Occidente. Casa de las Américas 98 , pp. 36-57.

GARCÍA CANCLINI, N. 1995. Narrar la multiculturalidad. In: García Canclini, N. Consumidores y Ciudadanos. Conflictos multiculturales de la globalización. México: Grijalbo, pp. 91-106.

GIDDENS, A. 1990. Modernity and Self-Identity. Self and Society. Apud: Hall, S. The

Local and the Global: Globalization and Ethnicity. In: Anthony King (ed.), Culture, Globalization and the World System. Londres: MacMillan, pp.19-39.

HALL, S. 1990. Old and New Identities, Old and New Ethnicities. In: A. King (ed.) Culture, Globalization and the World System. Londres: MacMillan, pp. 41-68

MARINI, M. / MILLÁN, M. 1994. La teoría social latinoamericana. México: El Caballito de Batalla, 2 tomos.

MEGGERS, B. J. 1978. Prólogo à Edição Norte-Americana. In: Darcy Ribeiro. $O$ processo Civilizatorio. Petrópolis: Vozes, pp. 15-20.

MIGNOLO, W. D. 1993. Colonial and Poscolonial Discourses: Cultural Critique or Academic Colonialism? Latin American Research Review 28: 120-131. 1996. Herencias coloniales y teorías poscoloniales. In: B. González Stephan (ed.) Cultura y Tercer Mundo. Cambios en el saber académico. Caracas: Nueva Sociedad, pp. 99-136.

1995. Human Understanding and (Latin) American Interests: The Politics and Sensibilities of Geocultural Locations. Poetics Today 16/1, pp. 171-214. . (no prelo) Posoccidentalismo: las epistemologías fronterizas y el dilema de los estudios (latinoamericanos) de área. Revista Iberoamericana.

MORLEY, D. / CHEN, K.-H. 1996. Stuart Hall: Critical Dialogues in Cultural Studies.

London: Routldege.

NANDY, A. 1995. The Savage Freud: The First non-Western Psychoanalyst and the

Politics of Secret Selves. In: Colonial India. The Savage Freud and Others Essays on Possible and Retrievable Selves. Princeton: Princeton U. Press: 81-144.

PLETSCH, C. 1981. Three Worlds, or the Division of Social Scientific Labor, circa 1950 - 1975. Comparative Studies in Society and History 23/24, pp.565-590.

PRAKAS, G. 1990. Writing Post-Orientalist Histories of the Third-World: Perspectives from Indian Historiography. Comparative Study of Society and History 32/2, pp. 383-408.

RIBEIRO, D. 1978 (1968) O processo civilizatório. Etapas da evolução socio-cultural. 
Petrópolis: Vozes.

1992 (1968) Las Américas y la civilización. Proceso de formación y causas del desarrollo desigual de los pueblos americanos. Caracas: Biblioteca Ayacucho. SAID, E. 1978. Orientalism. New York: Vintage Book, 1978.

SONNTAG, H. R. 1978. Epílogo à edição alemã. In: D. Ribeiro. O processo civilizatório Petrópolis: Vozes, pp. 203-216.

The Late Modern Age. 1991. Stanford: Stanford U. Press.

VEZZETTI, H. 1993. Psychoanalisis in the culture of Buenos Aires: The Thirties. Dispositio 45, pp. 141-157.

WALLERSTEIN, I. et. al. 1996. Open de Social Sciences. Report of the Gulbenkian Commission on the Restructuring of the Social Sciences. Stanford: Stanford U.Press. 